\title{
Land transformations in Hanyang city of Wuhan in Central China 2001-2011 \\ Yan $\mathrm{Li}^{1, \mathrm{a}}$
}

${ }^{1}$ Guoding Road No.777,Shanghai University of Finance and Economics, Shanghai, China 200433

adjna-liyan@foxmail.com

Keywords Land transformation; Exploratory Spatial Data Analysis; Hanyang; China

Abstract. Using the case of Hanyang, this paper provides new evidence of land transformation in China. Together with massive non-urban to urban land transformations, there is an increasing proportion of land being converted from urban to non-urban uses. The results of exploratory spatial data analysis demonstrate significant differences in their spatial distributions and clustering. It reveals that both ways of land transformations are being driven by the large-scale investment made by the public sectors and a growing private sector.

\section{Introduction}

The unprecedented land transformations in Chinese cities have drawn substantial research attentions. Exisiting literature concentrates on urban land expansion, i.e. land conversion from agricultural/natural vegetation to urban land (e.g. Li and Yeh 2004; Seto and Fragkias 2005; Tan, Li et al. 2005). However, as urban sustainability becomes a central issue in political and academic agendas, limiting urban sprawl and protecting natural zones have been increasingly incorporated in planning practices. Land development in Chinese cities has increasingly become a mixed process of urbanization and natural amenity development. Understanding this process is essential for urban planners and policy makers, and is the focus of this paper.
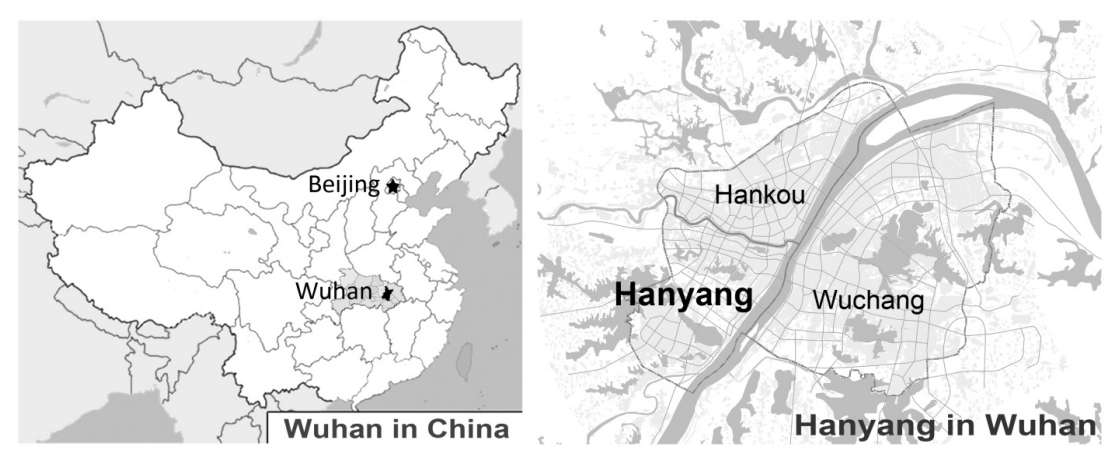

Figure 1: The location of Hanyang in Wuhan and in China

Hanyang is chosen for this research for two reasons. First, it is located in Wuhan in Central China (Figure 1), one of the fastest growing regions in China yet relatively unexplored in urban studies. Second, Hanyang is undergoing extensive land transformations, as its urban development focus has shifted from industries to historical and natural amenities.

Spatial analysis methods are used to quantify and analyze land use changes over time and space. The results show significant dynamics and different trendencies in the two different types of land transformations, which can be utilized for planners and policy makers to make more informed plans.

\section{Data and Methods}

Land use data of 2001, 2005, 2008 and 2011 are acquired from the Information Center of Wuhan Planning Bureau in the format of shapefile. A 500*500 meter grid is created to represent neighborhood scale. The share of transformed land (both ways separately) in each cell is calculated, as the input value for Exploratory Spatial Data Analysis, Moran's I coefficients developed by Anselin (1995). It assesses 
if cells with similar locations have shared similar (or distinctive) proportion of land transformed. Its values range between -1 (perfect dispersion) and 1 (perfect clustering); 0 indicates a completely random spatial distribution. To identify local clusters of positive or negative spatial autocorrelations, we further calculate the Local Indicators of Spatial Association (LISA) and produced LISA maps, visualizing four categories of local spatial association. The High-High $(\mathrm{HH})$ units represent concentrations of cells with high share of transformed land, while the Low-Low (LL) units represent concentrations of low shares. The High-Low (HL) and Low-High (LH) units represent the areas experienced significantly different land use changes with their neighbours.

\section{Analyzing Urban Land Use Change in Hanyang, 2001-2011}

Spatial Distribution of Land Use Change. To better understand and interpret the results, the entire study area is classified into three categories: 1) urban core, 2) suburb and 3) outskirts. Table 1 presents the statistical results of urban land growth over time in Hanyang. Overall the extent of urban land increased 28\% from 2001 to 2011. Not surprisingly, the growth in urban core during the ten years is negligible. By contrast, the amount of urban land growth in suburbs and outskirts is staggering, more than a hundred times of the urban core. Both have increased more than $30 \%$ in ten years' time.

Table 1: Statistics of urban land in Hanyang, 2001-2011

\begin{tabular}{lcccccc}
\hline & \multicolumn{2}{c}{ Urban land area } & & \multicolumn{2}{c}{ Urban land growth } \\
& 2001 & 2005 & 2008 & 2011 & ha & $\%$ \\
\hline Urban core & 1786 & 1803 & 1787 & 1798 & 13 & $1 \%$ \\
Suburbs & 3567 & 3652 & 4359 & 4890 & 1323 & $37 \%$ \\
Outskirt & 4428 & 4603 & 5201 & 5851 & 1423 & $32 \%$ \\
Total & 9781 & 10058 & 11347 & 12539 & 2759 & $28 \%$ \\
\hline
\end{tabular}
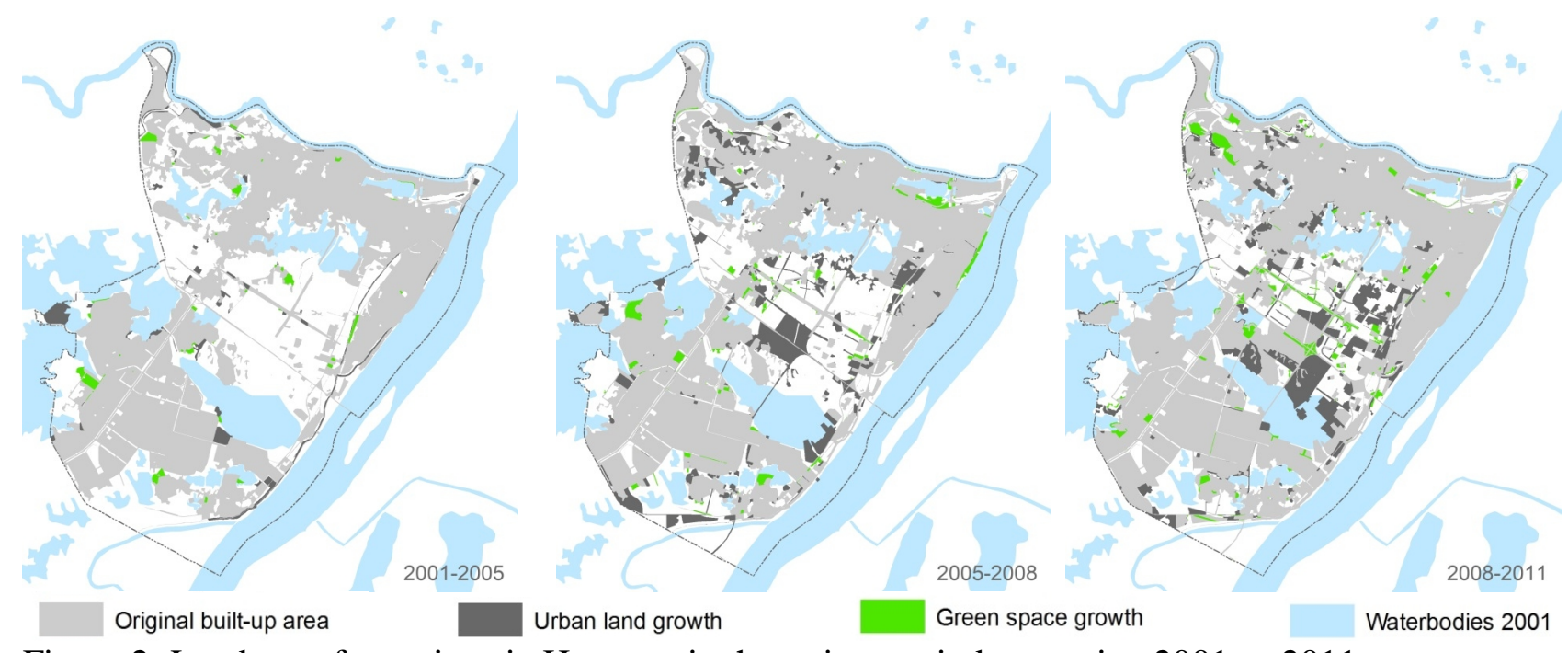

Figure 2: Land transformations in Hanyang in three time periods spanning 2001 to 2011

Maps in Figure 2 reveal that extensive growth of urban land took place after 2005. The first period, 2001-2005, has experienced limited land use transformations. From 2005 onwards, more roads are constructed in the central area between Moshui and Nantaizi Lake, connecting the urban core with suburb areas and the outskirts. Large scale urban land developments are found in Sixin area along the newly built roads, as it has been planned to be the new center of Hanyang city. Scattered urban land growths are also found on the edge of the city during 2005-2008, especially in the northwest and southeast corners. In the last period from 2008 to 2011, urban development takes place in Sixin and its adjacent areas, mostly in an infilling matter among existing built-up areas. It is also found that 
waterfront areas are gradually and consistently transformed into urban lands in all three periods, especially after 2005 around lakes at the edge of the city, which violates the master plan of Hanyang. The most visible example is the urban land growth to the north and to the east of Nantaizi Lake (Figure $2 \mathrm{~b}$ and 2c). From 2008 to 2011, green spaces are mostly found along the new roads in the new center areas. This indicates that natural amenities in central areas are better protected and developed.

Spatial patterns of land use change. Table 2 presents the global Moran's I coefficients calculated for both non-urban to urban and urban to non-urban land transformations in the three time periods. The growth of urban lands clearly shows a clustering tendency, as indicated by the increasing value of the Moran's I for all three periods. In contrast, urban to non-urban land transformation presents weak clustering in the first period, but shows an increasingly clustering trend in the following two periods.

Table 2: Global Moran's I coefficients for land transformations in Hanyang, 2001-2011

\begin{tabular}{llll}
\hline $\begin{array}{l}\text { Type of land } \\
\text { transformation }\end{array}$ & $2001-2005$ & $2005-2008$ & $2008-2011$ \\
\hline Non-urban to urban & $0.34 * * *$ & $0.38^{* * *}$ & $0.45^{* * *}$ \\
Urban to non-urban & $0.14 * *$ & $0.16^{* * *}$ & $0.24 * * *$ \\
\hline
\end{tabular}

Note: $* * *, * *$ and $*$ denote significance at the $0.1 \%, 0.5 \%$ and $1 \%$ levels respectively

Figure 3 presents the LISA maps of non-urban to urban land transformation. It is clear that in 2001-2005, HH areas are mostly small-scale and scattered in the edge areas of the city. From 2005 to 2008, more clusters emerged, with the largest one located in Sixin. This cluster grows larger and dominant in the third period. In all three periods, there are substantial LH values, mostly at the edge of $\mathrm{HH}$ areas. Fragmented land ownership, especially the rural lands owned collectively by villagers, may be one of the reasons for the fragmented patterns. Another possible reason is that new urban land development is mostly infilling among existing urban lands.
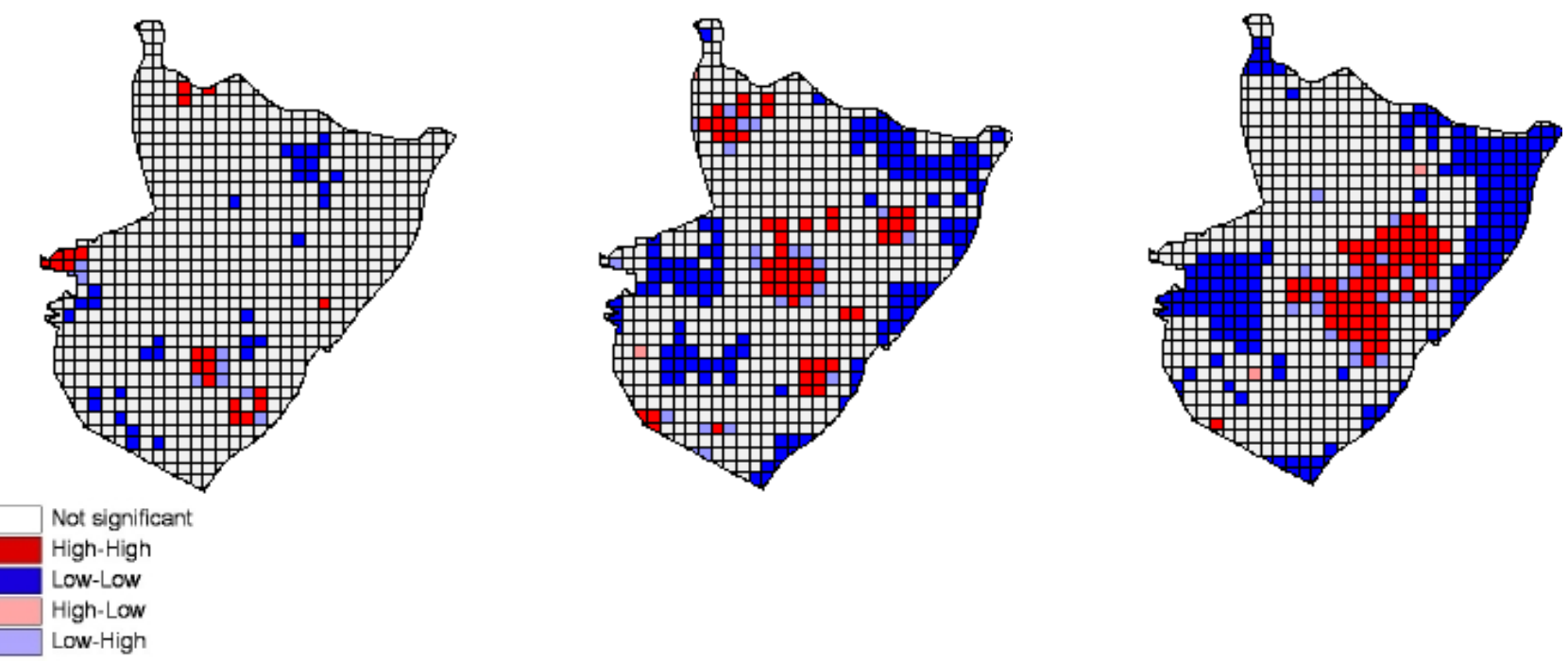
a. 2001-2005
b. $2005-2008$
c. $2008-2011$

Figure 3: LISA maps for non-urban to urban land transformation

Spatial clusters for non-urban land transformation are less prominent (Figure 4). In 2001-2005, HH and LH areas are highly mixed and scatter around the north and south, suggesting a fragmented distribution. In 2005-2008, the mixed HH and LH areas emerge around the lakes and Yangtze River, as part of the natural amenity development. In the third period, again new HH and LH mixed areas emerge, at the northwest corner as well as the Sixin area. These patterns suggest that urban to 
non-urban land transformations are geographically scattered, highly fragmented, and completed at a small spatial scale and in short time.

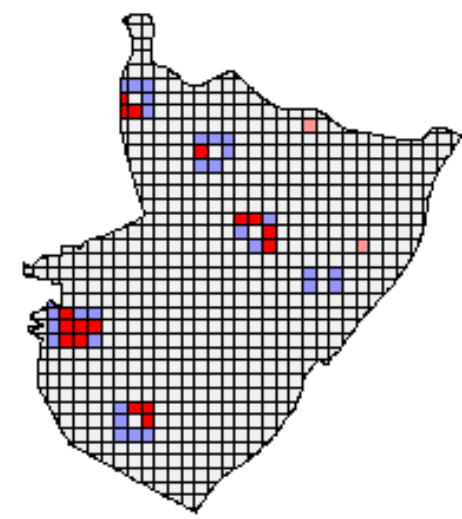

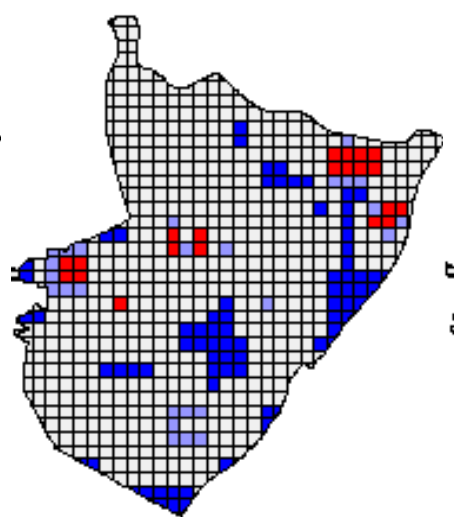

b. $2005-2008$

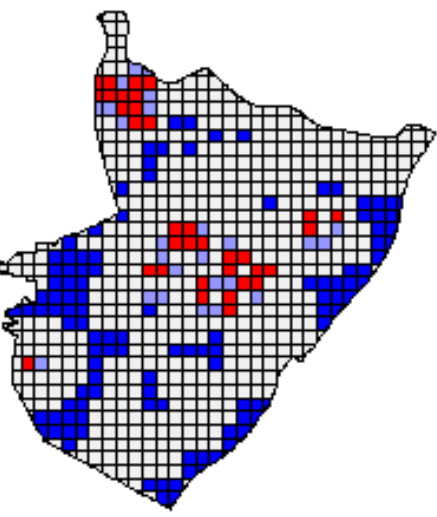

c. $2008-2011$ a. 2001-2005

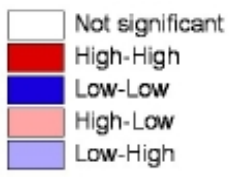

Figure 4: LISA maps for urban to non-urban land transformation

\section{Discussion and Conclusion}

The results of this paper demonstrate three typical development trends in Chinese cities. First, large scale urban land development occurs in suburbs and outskirts, mainly directed by policies. Second, new urban lands are accessibility sensitive, therefore are typically along the transportation network. Third, natural and historical amenities are more appreciated and thus better protected and developed in urban core than remote areas of the city. These findings are partly consistent with empirical findings of urban land growth in cities, that new urban development often takes place in targeted development zones, and mostly along transportation networks (Kennedy 2007; Xu, Liu et al. 2007; Han, Hayashi et al. 2009). It also adds to the literature that urban to non-urban land transformations are emerging due to the increasing demand for natural amenities in urban areas.

It is expected that urbanization in China will continue to grow in the coming years (Jiang, Deng et al. 2013). New trends of land use transformations, spatial clustering, and increasing demand for natural amenity in urban areas have largely been driven by large-scale investment by public agencies and a growing private sector, the behavior of which has not been internalized into municipal planning processes. Planning and policy making must take into account the new trends and new measures should be developed to better facilitate resource management and allocation.

\section{References}

[1] Anselin, L. (1995). "Local Indicators of Spatial Association - LISA." Geographical Analysis 27(2): 93-115.

[2] Han, J., Y. Hayashi, et al. (2009). "Application of an integrated system dynamics and cellular automata model for urban growth assessment: A case study of Shanghai, China." Landscape and Urban Planning 91(3): 133-141.

[3] Jiang, L., X. Deng, et al. (2013). "The impact of urban expansion on agricultural land use intensity in China." Land Use Policy 35: 33-39.

[4] Kennedy, L. (2007). "Regional industrial policies driving peri-urban dynamics in Hyderabad, India." Cities 24(2): 95-109.

[5] Li, X. and A. G.-O. Yeh (2004). "Analyzing spatial restructuring of land use patterns in a fast growing region using remote sensing and GIS." Landscape and Urban Planning 69(4): 335-354.

[6] Seto, K. C. and M. Fragkias (2005). "Quantifying Spatiotemporal Patterns of Urban Land-use Change in Four Cities of China with Time Series Landscape Metrics." Landscape Ecology 20(7): 871-888. 
[7] Tan, M., X. Li, et al. (2005). "Urban land expansion and arable land loss in China — a case study of Beijing-Tianjin-Hebei region." Land Use Policy 22(3): 187-196.

[8] Xu, C., M. Liu, et al. (2007). "The spatiotemporal dynamics of rapid urban growth in the Nanjing metropolitan region of China." Landscape Ecology 22(6): 925-937. 